\title{
Diagnostic \& Prognostic Evaluation of Urinary Abnormalities for Malaria Associated Kidney Disease
}

\author{
Authors \\ Dr Samir Govil $^{1 *}$, Dr Parag Chandra ${ }^{2}$, Dr Richa Giri ${ }^{3}$ \\ ${ }^{1}$ Associate Prof., Deptt of Medicine, GSVM Medical College Kanpur \\ ${ }^{2}$ Resident, Deptt of Medicine, GSVM Medical College Kanpur \\ ${ }^{3}$ Prof \& Head, Deptt of Medicine, GSVM Medical College Kanpur \\ *Corresponding Author \\ Dr Samir Govil MD, DNB (Nephrology) \\ 372 Vikas Nagar Kanpur 208024, Uttar Pradesh, India
}

\begin{abstract}
Background: Malaria is a multi-organ illness due to plasmodium species and involves the brain, liver and kidneys ${ }^{1}$. Urinary abnormalities are frequently found in malaria patients and may be associated with a wide spectrum of renal injury including chronic kidney disease.

Objective: To correlate urinary abnormalities in malaria patients with the occurrence of kidney disease and its clinical outcomes.

Methods: A prospective study was conducted in 458 patients of confirmed malaria, to measure urinary abnormalities including proteinuria and hematuria at presentation, and correlate them with kidney injury reflected by a falling creatinine clearance rate $<60 \mathrm{mg} / \mathrm{m}^{2}$. Patients were treated using standard care pathways and followed for 6 months after discharge from hospital. Persistence or resolution of kidney injury was identified using the parameters mentioned above. Statistical analyses were done using standard tests and a ' $p$ ' value $<0.05$ was considered significant.

Results: Urinary abnormalities were found in 230 of 458 (50.21\%) patients, and were equally divided between both plasmodium species. Older patients (age>30 years) were more frequently affected, and there wer no gender difference in urinary abnormalities in either species infestation. The average number of dialysis sessions was also similar, while hospital length of stay was significantly greater at 7.29+/-2.94 days in the P. falciparum patients vs. 6.18+/-3.11 days for P. vivax ( $p<0.05 .198 / 230) .198$ patients survived and 6 months follow up was completed in 142 patients. Complete resolution of kidney disease occurred in 70/142 patients, while 72/142 had residual disease progressing to chronic kidney disease (CKD). There was a significant correlation between urinary abnormalities in malaria patients and renal disease at follow up ( $p<0.05)$, which had a negative predictive value of $57.98 \%$ with a sensitivity of $20.22 \%$ and specificity $69.50 \%$.

Conclusion: Presence and persistence of urinary abnormalities in patients with malaria may be early markers of renal injury. Absence of these urinary abnormalities at follow up suggests resolution of renal insult associated with malaria and low likelihood of progression to CKD.

Keywords: urinary abnormalities, kidney disease, and malaria.
\end{abstract}

\section{Introduction}

Malaria is a vector borne illness caused by the parasite species Plasmodium and spread by the female Anopheles mosquito ${ }^{1}$. It is endemic in warm humid tropical and subtropical countries, and affects both children and adults. Fever is the 
initial presentation and may be intermittent or continuous. It rises rapidly and is characteristically associated with shaking chills and rigor, profuse sweating, headache, muscle and joint pain, nausea, vomiting and loss of appetite. Other symptoms may include diarrhea, skin rash, abdominal pain and features of upper respiratory tract infection ${ }^{2}$.

Severe malaria may present as altered consciousness, convulsions, respiratory distress, renal impairment ${ }^{3}$, jaundice, abnormal bleeding or shock $^{4}$. Laboratory investigations may reveal anaemia, hypoglycemia, acidosis, raised creatinine, hyperbilirubinaemia and hyperparasitaemia. Several factors influence the progression of uncomplicated malaria to severe malaria, including plasmodium species, age and gender of the patient, host immunity, parasitic load, and availability of timely and effective treatment. In recent years hepatic and renal complications have replaced cerebral malaria as the predominant manifestation of severe malaria ${ }^{8}$. Urinary abnormalities like albuminuria (71.2\%), pyuria $(53.8 \%)$, hematuria $(45 \%)$ and granular casts $(71.4 \%)$ have been demonstrated in patients with malaria ${ }^{7}$. Proteinuria and hematuria have been shown to be predictors of end-stage renal disease in mass screening of population ${ }^{5}$. However, urinary abnormalities may be present in malaria even in the absence of renal disease.

Malaria associated renal impairment has a wide spectrum, including asymptomatic proteinuria or hematuria, acute glomerulonephritis, acute kidney injury, nephritic or nephrotic syndrome ${ }^{6}$ and chronic kidney disease. In India, four species of Plasmodium have been identified to cause malaria. $P$. vivax infection occurs in $60-75 \%$ of patients while $P$. falciparum accounts for $35-40 \%$ of malaria cases ${ }^{8}$. The latter is implicated in severe malaria and is thought to be more often associated with renal dysfunction. However, $P$. vivax is increasingly being seen to cause kidney injury. Other species of plasmodium are less common and their contribution to cases of severe malaria is relatively low.
We attempted to study the correlation of urinary abnormalities with kidney injury in malaria patients infected with $P$. falciparum or $P$. vivax. Our objective was to evaluate proteinuria and hematuria as potential diagnostic and prognostic predictors of kidney disease and its outcomes.

\section{Methods}

A prospective study was conducted in the KPS Institute of Medicine, GSVM Medical College, Kanpur. A total of 458 adult patients of $P$. falciparum and $P$. vivax malaria presenting between January 2016 and October 2018 were included in the study. Diagnosis of malaria was established using a thick and thin peripheral blood smear stained with Leishman's stain, and the rapid malarial antigen card test ${ }^{2}$.

\section{Inclusion Criteria}

1. Confirmed diagnosis of malaria

2. Urinary abnormalities with or without decline of age and weight adjusted creatinine clearance below normal

\section{Exclusion Criteria}

1. Patients $<18$ years of age

2. Mixed infections with more than one plasmodium species

3. Known history or clinical/laboratory evidence of kidney disease

4. Pregnant women

All patients underwent a detailed history and clinical examination including recording of vital parameters, and their blood and urine samples were collected at presentation. A complete blood count (CBC), renal function test (RFT), serum electrolytes (SE), liver function test (LFT), random plasma glucose (RPG) and urinalysis were performed in our standardized laboratory. Triple screening for HIV, HBsAg and HCV was done for all patients using the card test. A baseline creatinine clearance $(\mathrm{CrCl})$ was calculated for each patient using the Cockcroft-Gault equation": Creatinine Clearance $=\operatorname{Sex} *((140-$ Age $) /$ (Serum Creatinine)) *(Weight / 72).

Malaria treatment was given using oral or parenteral medication as indicated, according to 
the 2014 guidelines provided by the National Institute of Malaria Research ${ }^{2}$. Renal replacement therapy was offered to patients with features of severe renal injury not responding to conservative treatment. These included severe hyperkalemia, intractable acidosis, fluid overload or oliguria. A 6 months follow-up for all patients was conducted after discharge and blood tests and urinalysis were repeated at this time.

\section{Statistical Analysis}

Statistical analyses were conducted using standardized tests. Difference in proportions was compared by chi-square test (significant ' $p$ ' value $<0.05)$. The sensitivity, specificity, positive and negative predictive values were calculated, along with relative risk for malaria patients developing kidney disease in the presence of urinary abnormalities.

\section{Results}

The data from 458 patients of malaria including 190 (41.5\%) of P. falciparum and 268 (58.5\%) of $P$. vivax malaria are shown below.

Table 1: Presence of urinary abnormalities (proteinuria and/or hematuria) in malaria patients.

\begin{tabular}{|l|c|c|c|c|}
\hline & $\begin{array}{c}\text { Urinary } \\
\text { Abnormalities }\end{array}$ & Present & Absent & Total \\
\hline $\begin{array}{l}\text { Malaria } \\
(\mathrm{n}=458)\end{array}$ & Yes & $\begin{array}{c}230 \\
(50.21 \%)\end{array}$ & $\begin{array}{c}228 \\
(49.79 \%)\end{array}$ & $\begin{array}{c}458 \\
(100 \%)\end{array}$ \\
\hline
\end{tabular}

Almost half of the patients of malaria in the study had urinary abnormalities, while the remainder had normal urine routine and microscopy results.

Table 2: Number of patients with urinary abnormalities according to species of the malarial parasite.

\begin{tabular}{|l|c|c|c|}
\hline \multirow{2}{*}{$\begin{array}{l}\text { Type } \\
\text { Malaria } \Downarrow\end{array}$} & \multicolumn{2}{|c|}{ Urinary Abnormalities $\Rightarrow$} & \\
\cline { 2 - 4 } & $\begin{array}{c}\text { Present }(\%) \\
(\mathrm{n}=230)\end{array}$ & $\begin{array}{c}\text { Absent }(\%) \\
(\mathrm{n}=228)\end{array}$ & $\begin{array}{c}\text { Total }(\%) \\
(\mathrm{n}=458)\end{array}$ \\
\hline P. falciparum & $101(43.91)$ & $89(39.04)$ & $190(41.48)$ \\
\hline P. vivax & $129(56.09)$ & $\begin{array}{c}139 \\
(60.96)\end{array}$ & $268(58.51)$ \\
\hline
\end{tabular}

$P$. vivax malaria was slightly more common than

$P$. falciparum, but the percentage of malaria patients presenting with urinary abnormalities was statistically similar.

Tables 3 and 4 below show the appearance of urinary abnormalities in $P$. falciparum and $P$. vivax malaria patients respectively, as a function of age.

Table 3: Age distribution in $P$. falciparum malaria patients

\begin{tabular}{|l|l|l|l|}
\hline & \multicolumn{2}{|l|}{ Urinary Abnormalities $\Rightarrow$} & \\
\cline { 1 - 2 } $\begin{array}{l}\text { Age (years) } \\
\Downarrow\end{array}$ & Present (\%) & Absent (\%) & Total (\%) \\
\hline$<30$ & $9(8.91)$ & $18(20.22)$ & $27(14.21)$ \\
\hline $31-60$ & $48(47.52)$ & $39(43.82)$ & $87(45.79)$ \\
\hline$>60$ & $44(43.57)$ & $32(35.96)$ & $76(40)$ \\
\hline Total & 101 & 89 & 190 \\
\hline
\end{tabular}

$\mathrm{X}^{2}=5.088, \mathrm{p}=0.67854$ (not significant), $\mathrm{Df}=2$

Table 4: Age distribution in $P$. vivax malaria patients

\begin{tabular}{|l|c|c|c|}
\hline & \multicolumn{2}{|c|}{ Urinary Abnormalities $\Rightarrow$} & \multirow{2}{*}{ Total $(\%)$} \\
\hline $\begin{array}{l}\text { Age (years) } \\
\Downarrow\end{array}$ & Present (\%) & Absent $(\%)$ & \\
\hline$<30$ & $5(3.87)$ & $14(10.07)$ & $19(7.00)$ \\
\hline $31-60$ & $66(51.67)$ & $73(52.52)$ & $139(51.86)$ \\
\hline$>60$ & $58(44.96)$ & $52(37.41)$ & $110(41.05)$ \\
\hline Total & 129 & 139 & 268 \\
\hline
\end{tabular}

$\mathrm{X}^{2}=0.1454, \mathrm{p}=0.1015$ (not significant), $\mathrm{Df}=2$

Three age groups were compared including young patients $<30$ years of age, adults $31-60$ years and older patients $>60$ years. There was no statistically significant difference in the occurrence of urinary abnormalities between the three age groups. However, patients younger than 30 years had lower, while patients older than 30 years had a higher incidence of urinary abnormalities in malaria from both species $(\mathrm{p}<0.05)$.

Table 5: Gender distribution of patients according to Plasmodium species

\begin{tabular}{|l|c|c|}
\hline $\begin{array}{l}\text { Plasmodium } \\
\text { species } \Rightarrow \\
\text { Patient gender } \Downarrow\end{array}$ & $\begin{array}{c}P . \text { falciparum } \\
(\mathrm{n}=101)\end{array}$ & $\begin{array}{l}P . \text { vivax } \\
(\mathrm{n}=129)\end{array}$ \\
\hline Male & 57 & 73 \\
\hline Female & 44 & 56 \\
\hline
\end{tabular}

Urinary abnormalities were commoner in males $(56.5 \%)$ than females $(43.5 \%)$, but the gender ratio (females:males) was similar between the 
Plasmodium species. (P. falciparum 1:1.3 and $P$. vivax 1:1.3)

The spectrum of urinary abnormalities and clinical kidney disease in patients with malaria is shown below in tables $6 \& 7$, respectively.

Table 6: Frequency of urinary abnormalities and features of kidney disease:

\begin{tabular}{|l|l|l|}
\hline & P. falciparum & P. vivax \\
\hline Proteinuria (only) & $38(38 \%)$ & $31(24 \%)$ \\
\hline Proteinuria + Haematuria & $15(15 \%)$ & $10(8 \%)$ \\
\hline Decreased urine output & & \\
\hline - Oliguria $(<500 \mathrm{ml} / 24 \mathrm{~h})$ & $61(60 \%)$ & $49(38 \%)$ \\
\hline - Anuria $(<50 \mathrm{ml} / 24 \mathrm{~h})$ & $18(18 \%)$ & $9(7 \%)$ \\
\hline
\end{tabular}

Proteinuria alone was the commonest finding on urinalysis, while that associated with haematuria was relatively less frequent. The majority of patients with kidney disease presented with oliguria, although anuria was not infrequent. These results were comparable in infections due to either plasmodium species ( $\mathrm{p}=\mathrm{NS}$ ).

Table 7: Clinical features of malaria associated kidney disease

\begin{tabular}{|l|c|c|}
\hline Clinical Features & P. falciparum & $P$. vivax \\
\hline Proteinuria & $18(17.9 \%)$ & $15(11.6 \%)$ \\
\hline Decreased urine output & $56(55.6 \%)$ & $14(10.8 \%)$ \\
\hline Metabolic acidosis & $19(18.8 \%)$ & $15(11.6 \%)$ \\
\hline Hyperkalemia & $16(15.8 \%)$ & $16(12.4 \%)$ \\
\hline Volume overload & $16(15.8 \%)$ & $17(13.2 \%)$ \\
\hline Uremic encephalopathy & $4(4 \%)$ & $3(2.3 \%)$ \\
\hline
\end{tabular}

Decreased urine output with or without other clinical findings was the most frequent complaint in patients of $P$. falciparum malaria. Those presenting with other features like isolated proteinuria, hyperkalaemia, metabolic acidosis and fluid overload were similarly distributed across both groups, and uremic encephalopathy was uncommon.

\section{Clinical Intervention}

Treatment of malaria-associated kidney disease was done according to guidelines ${ }^{2}$, including parenteral administration of anti malarial drugs, antipyretics, antibiotics and anticonvulsants, and supportive measures like maintaining fluid and electrolyte balance. Laboratory services were available along with facilities for intensive care, oxygen supply, mechanical ventilation, blood transfusion, and dialysis and specialized nursing care.

\section{Conservative}

1. Artemisin based therapy: artesunate 2.4 $\mathrm{mg} / \mathrm{kg}$ intravenously after dilution as recommended

2. Antibiotics (where indicated)

3. Intravenous fluids

4. General supportive treatment

\section{Conservative (as above) + Intravenous}

1. Low dose Frusemide - 20 mg BD

2. High dose Frusemide - $60 \mathrm{mg}$ TID

\section{Renal replacement therapy}

Criteria for hemodialysis:

1. Decreased urine output (urine output $<500$ $\mathrm{ml} / 24$ hours)

2. Severe acidosis $(\mathrm{pH}<7.20)$

3. Hyperkalemia $(>6 \mathrm{meq} / \mathrm{L})$

4. Persistently declining creatinine clearance (>30\% of baseline/24 hours)

\section{Rationale for hemodialysis}

To allow the kidney to recover from the malarial pathophysiological insult, including:

1. Correction of acidosis and electrolyte imbalance

2. Increased clearance of uremic toxins

3. Treatment of fluid overload

Table 8 below, shows the number of patients of malaria with kidney disease, which required renal replacement therapy and the average number of hemodialysis sessions, average length of stay, and in hospital mortality.

Table 8: Renal replacement therapy for kidney disease in malaria

\begin{tabular}{|l|c|c|}
\hline Outcome & $\begin{array}{c}\text { P. falciparum } \\
(\mathrm{n}=101)\end{array}$ & $\begin{array}{c}\text { P. vivax } \\
(\mathrm{n}=129)\end{array}$ \\
\hline \# Of patients for dialysis & $25(24.7 \%)$ & $26(20.2 \%)$ \\
\hline \# Of dialysis sessions & $3.38 \pm 1.26$ & $3.24 \pm 1.21$ \\
\hline Length of stay (days) & $7.29 \pm 2.94$ & $6.18 \pm 3.11^{+}$ \\
\hline In hospital mortality & $11(10.9 \%)$ & $17(13.2 \%)$ \\
\hline
\end{tabular}

Patients in the $P$. vivax group fared marginally better than $P$. falciparum in the requirement and length of dialysis, and in-hospital mortality, although this difference was not statistically 
significant. The length of stay, however, was longer for $P$. falciparum than for $P$. vivax affected patients $(\mathrm{p}<0.05)$.

Table 9: Patients with malaria associated urinary abnormalities and kidney disease

\begin{tabular}{|l|c|c|c|}
\hline Urinary Abnormalities $\Rightarrow$ & Present & Absent & Total \\
\cline { 1 - 2 } $\begin{array}{l}\text { Impaired renal function } \\
\text { (age adjusted eGFR<60 } \\
\text { ml/min) } \downarrow\end{array}$ & & & \\
\hline Present & 18 & 43 & 61 \\
\hline Absent & 71 & 98 & 169 \\
\hline Total & 89 & 141 & 230 \\
\hline
\end{tabular}

$\mathrm{X} 2=2.941, \mathrm{P}<0.05$ (significant)

The above table shows the frequency of kidney disease, reflected by a falling creatinine clearance $<60 \mathrm{ml} / \mathrm{min}$, as a function of urinary abnormalities in malaria patients. The Mantel Haenszel chisquare test shows a statistically significant association between these two variables. The overall risk of developing kidney disease in malaria is $38.70 \%$, while a relative risk of 0.69 predicts the likelihood of renal sparing in the absence of urinary abnormalities. The sensitivity of the association is low at $20.22 \%$ while the specificity is moderate at $69.50 \%$, and the positive predictive value is $29.50 \%$. However, a negative predictive value of $57.98 \%$ makes it a favourable indicator of preserved kidney function, in the absence of urinary abnormalities in patients with malaria.

Of the 230 malaria patients with kidney disease 198 survived and were followed up for 6 months after discharge from the hospital. At the end of the follow up period, data was available for 142 patients. The following table shows the results:

Table 10: Patients at 6 months follow up

\begin{tabular}{|l|c|c|c|}
\hline & $\begin{array}{c}\text { Normal - } \\
(\mathbf{n = 7 0}) \\
\mathrm{eGFR}>60 \\
\mathrm{mg} / \mathrm{min}\end{array}$ & $\begin{array}{c}\text { CKD - } \\
\mathbf{N D D}^{+} \\
(\mathbf{n = 6 2})\end{array}$ & $\begin{array}{c}\text { CKD }-\mathbf{D D}^{++} \\
(\mathbf{n = 1 0})\end{array}$ \\
\hline $\begin{array}{l}\text { CrCl (eGFR) } \\
\mathbf{m g} / \text { min }\end{array}$ & $72+/-8$ & $45+/-16$ & $12+/-7$ \\
\hline Proteinuria & $<0.1 \mathrm{~g} / \mathrm{day}$ & $>0.5 \mathrm{~g} / \mathrm{day}$ & $0.1-0.5 \mathrm{~g} / \mathrm{day}$ \\
\hline $\begin{array}{l}\text { Proteinuria + } \\
\text { Haematuria }\end{array}$ & $<4 \mathrm{RBC} / \mathrm{hpf}$ & $\begin{array}{c}5-10 \\
\mathrm{RBC} / \mathrm{hpf}\end{array}$ & $5-10 \mathrm{RBC} / \mathrm{hpf}$ \\
\hline
\end{tabular}

${ }^{+} \mathrm{NDD}$ - non-dialysis dependent (eGFR range $15-60 \mathrm{mg} / \mathrm{m} 2$ ),

${ }^{++} \mathrm{DD}$ - dialysis dependent (eGFR range $<15 \mathrm{mg} / \mathrm{m} 2$ )

Of the 142 patients with a 6 months follow-up, 72 patients had residual renal dysfunction of which
62 patients were managed conservatively, and 10 developed chronic kidney disease requiring haemodialysis. 70 patients recovered completely and regained normal kidney function with no urinary abnormalities or signs of renal dysfunction.

\section{Discussion}

Malaria is a common tropical illness spread widely across the Indian subcontinent. It is often associated with a single or multiple system dysfunctions, leading to significant morbidity and mortality. $P$. falciparum is known to cause more severe disease, associated with anaemia, hemodynamic compromise, severe oliguria, neurological dysfunction and sepsis. P. vivax on the other hand, is complicated by liver injury, blood dyscrasias, splenomegaly and sepsis.

In the last decade or so the pattern of malarial complications in both plasmodium species appears to have changed, and a higher incidence of sepsis, electrolyte abnormalities, acid base disturbances and kidney injury are seen. The reason for this is not clear but the outcomes in these complicated malaria patients are poor.

The malarial parasite can cause direct kidney injury, inflammation and consequent glomerulonephritis. Renal complications may also result from altered host immune response to the parasite, hyperbilirubinaemia, electrolyte imbalance and hemodynamic compromise. Acute kidney injury including acute tubular necrosis and acute interstitial nephritis may require hemodialysis, and occasionally lead to chronic kidney disease ${ }^{11}$.

Patients presenting with fever may have urinary abnormalities like proteinuria and hematuria as a part of the systemic illness, and the renal function remains normal (age and weight matched $\mathrm{CrCl}>60$ ). In severe malaria kidney affectation may manifest as a spectrum of abnormalities, including rising creatinine, metabolic acidosis, hyperkalemia and oliguria ${ }^{12}$. The risk factors for renal injury includes older age, hypotension, 
superadded infection, altered sensorium and decreased urine output ${ }^{13}$.

Hyperparasitemia and host immune response can both cause renal tubular damage. Low perfusion pressure due to hypovolemia and shock adds to the insult and may lead to acute tubular injury, while a damaged endothelium in the capillaries causes proteinuria and hematuria, which in turn may further occlude the microcirculation. The resultant release of inflammatory cytokines like endothelins, interleukins and thromboxane, leads to formation and deposition of immune complexes, causing glomerulonephritis or acute interstitial nephritis. Cortical necrosis is an uncommon but usually irreversible manifestation of kidney disease in malaria ${ }^{11}$.

It has been shown that one third of patients with malaria associated kidney injury have normal age matched creatinine clearance at presentation. Neutrophil gelatinase-associated lipocalin (NGAL) and kidney injury molecule-1 (KIM-1) are early biomarkers for acute kidney injury, and can be detected earlier than rise in creatinine levels ${ }^{14}$. Early diagnosis and prompt initiation of renal replacement therapy is shown to be associated with improved clinical outcome and survival $^{15}$.

\section{Limitations}

1) The study was conducted at a teaching hospital where the sickest patients are admitted. The occurrence of asymptomatic urinary abnormalities in patients with fever and malaria might be significantly higher.

2) Kidney injury could not be characterized accurately as only clinical and laboratory data were collected. A kidney biopsy could provide deeper insight into the nature of kidney disease, improving our diagnostic, therapeutic and prognostic capabilities.

3) Follow up data could not be captured in its entirety, as some patients were lost to follow up.

\section{Conclusion}

A significant number of malaria patients present with urinary abnormalities and develop complications needing specialized care in a hospital. Renal involvement poses a substantial disease burden in severe malaria, and most patients respond well to conservative therapy and supportive measures. Renal replacement therapy may be required in half to two thirds of the patients with severe kidney disease, and treatment outcomes are good with a reasonable likelihood of regaining complete renal function.

\section{Recommendations}

1) Primary care physicians most commonly diagnose malaria and its complications. They must maintain a high index of suspicion to detect signs and symptoms suggestive of severe disease, and refer to a well-equipped hospital to manage complications.

2) Baseline hematological and biochemical parameters like complete blood count, serum creatinine, electrolytes, should be monitored at presentation and followed through to recovery, as a diagnostic and prognostic tool for complications of malaria.

\section{References}

1. Sharma R, Dutta AK. Malaria and National Vector Borne Disease Control Programme. Indian J Pediatr. 2011 Dec;78(12):1527-35.

2. Guidelines for Diagnosis and Treatment of Malaria in India (Third Edition: July 2014)); National Vector Borne Disease Control Programme; National Institute of Malaria Research, New Delhi.

3. Elsheikha HM, Sheashaa HA. Epidemiology, pathophysiology, management and outcome of renal dysfunction associated with plasmodia infection. Parasitol Res. 2007;101:118390. 
4. Severe Malaria. 2014 WHO. Tropical Medicine and International Health 2014 September; Volume 19;Supple.(I):7-131.

5. K Iseki, C Iseki, Y Ikemiya, K Fukiyama. Risk of developing end-stage renal disease in a cohort of mass screening. Kidney Int 1996;49:800-805.

6. MM Rajapurkar. Renal involvement in malaria. Postgrad Med J 1996;40:132.

7. AO Karoum, BA Mohammed. Urine analysis in malaria in Kassala town, Eastern Sudan. Saudi J Kidney Dis Transpl 200;11:208-209.

8. D Rath, MC Sahu. The clinical features of complicated falciparum malarial nephropathy. J Taibah Univ Med Sc 2017; 12(2):110-114

9. EI Ugwuja, NC Ugwu. Abnormal findings on dipstick urinalysis of outpatients with malaria in Abakaliki, Nigeria. J Vector Borne Dis 48, December 2011:205-209.

10. Cockcroft DW, Gault MH. Prediction of creatinine clearance from serum creatinine. Nephron. 1976;16(1):31-41.

11. Naqvi R. Plasmodium vivax causing acute kidney injury: a foe less addressed. Pak J Med Sci. 2015;31:1472-5.

12. Naqvi R, Akhtar F, Ahmed E, Sheikh R, Bhatti S, Haider A, et al. Malarial acute kidney injury: 25 years experience from a center in an endemic region. $\mathrm{Br} \mathrm{J}$ Med Med Res. 2016;12:21471.

13. Win KK, Thanachartwet V, Wattanagoon Y, Jerraksuwan S, Ruangweerayut R, Desakorn V. Factors associated with acute renal failure in adults with severe falciparum malaria. Southeast Asian J Trop Med Public Health. 2012;43:1071-9.

14. van Wolfswinkel ME, Koopmans LC, Hesselink DA, Hoorn EJ, Koelewijn R, van Hellemond $J J$, et al. Neutrophil gelatinase-associated lipocalin (NGAL) predicts the occurrence of malaria-induced acute kidney injury. Malaria J. 2016;15:464.
15. Wang C, Lv LS, Huang H, Guan J, Ye Z, $\mathrm{Li} \mathrm{S}$, et al. Initiation time of renal replacement therapy on patients with acute kidney injury: a systematic review and meta-analysis of 8179 participants. Nephrology (Carlton). 2017;22:7-18. 